\title{
A statistical subgrid scale model for large eddy simulations
}

Rukiye KaraMine çağlar

Citation: AIP Conference Proceedings 1558, 318 (2013); doi: 10.1063/1.4825487

View online: http://dx.doi.org/10.1063/1.4825487

View Table of Contents: http://aip.scitation.org/toc/apc/1558/1

Published by the American Institute of Physics 


\title{
A Statistical Subgrid Scale Model for Large Eddy Simulations ${ }^{1}$
}

\author{
Rukiye Kara* and Mine Çağlar ${ }^{\dagger}$ \\ * Mimar Sinan Fine Arts University, Department of Mathematics, Istanbul, TURKEY \\ ${ }^{\dagger}$ Koc University, Department of Mathematics, Istanbul, TURKEY
}

\begin{abstract}
The energy spectrum plays a central role for modeling the subgrid stress term in filtered Navier-Stokes equations. Considering a truncated Gamma distribution for radius of eddies, the subgrid scale energy spectrum has been computed analytically. In this study, we develop a new subgrid stress model for representing the small scale effects in LES by defining the parameters of the energy spectrum.

Keywords: Stochastic flows; homogeneous turbulence; large eddy simulation; subgrid stress modeling.
\end{abstract}

PACS: 76F05,60E99

\section{INTRODUCTION}

In fluid dynamics, turbulence has chaotic and stochastic properties. The turbulence theory examines continuous change of eddy sizes. The fundamental equations are Navier-Stokes equations, which are derived from conservation of mass and momentum. In three-dimensional space, the smoothness and the existence of the solution of Navier-Stokes equations is an open problem. In order to find a numerical solution of these equations, all the time and space scales of the flow dynamics must be taken into account. In this case, the computations are very costly. For this reason, while some of the scales could directly be calculated with the numerical algorithms, the others should be modeled. In this study, a novel subgrid model will be examined for large eddy simulation (LES), which is one of the solution methods of Navier-Stokes equations. In LES, a filter is applied to Navier-Stokes equations. As a result of filtering the momentum equation, a nonlinear subgrid stress term is obtained and modeled for demonstrating the effect of the smaller scales. A random velocity field whose parameters can be estimated from real data will be used for modeling the subgrid stress term.

\section{LARGE EDDY SIMULATION}

The motion of fluid is described by Navier-Stokes equations. These equations arise from conservation of mass and momentum laws for a fluid. The general form of the equations of fluid motion is

$$
\begin{aligned}
\frac{\partial u_{i}}{\partial x_{i}} & =0 \\
\frac{\partial u_{i}}{\partial t}+u_{j} \frac{\partial u_{i}}{\partial x_{j}} & =-\frac{\partial p}{\partial x_{i}}+v \frac{\partial^{2} u_{i}}{\partial x_{j} \partial x_{j}}
\end{aligned}
$$

where $u_{i}$ is the flow velocity components, $p$ is the pressure and $v$ is the kinematic viscosity. A filtering operation separates large scales from the smaller ones by

$$
\overline{u(\mathbf{x})}=\int_{-\infty}^{\infty} G\left(x-x^{\prime}\right) u\left(x^{\prime}\right) d^{3} x^{\prime}
$$

\footnotetext{
${ }^{1}$ This work is supported by TUBITAK Project No.112T761
} 
where $G$ is the filter function that determines the size and structure of the small scales. If the filtering operation is applied to (1), the filtered Navier-Stokes equations are obtained as

$$
\begin{aligned}
\frac{\partial \bar{u}_{i}}{\partial x_{i}} & =0 \\
\frac{\partial \bar{u}_{i}}{\partial t}+\frac{\partial \bar{u}_{i} \bar{u}_{j}}{\partial x_{j}} & =-\frac{\partial \bar{p}}{\partial x_{i}}+v \frac{\partial}{\partial x_{j}}\left(\frac{\partial \bar{u}_{i}}{\partial x_{j}}+\frac{\partial \bar{u}_{j}}{\partial x_{i}}\right)-\frac{\partial \tau_{i j}}{\partial x_{j}}
\end{aligned}
$$

where $\tau_{i j}=\overline{u_{i} u_{j}}-\bar{u}_{i} \bar{u}_{j}$ is subgrid scale tensor and it must be modeled to represent the effect of unresolved scales. When the velocity field is expressed as the sum of resolved and unresolved (fluctuating) field by $u=\bar{u}+u^{\prime}$, subgrid stress tensor can be decomposed into three parts:

$$
\tau_{i j}=\overline{u_{i} u_{j}}-\bar{u}_{i} \bar{u}_{j}=L_{i j}+C_{i j}+R_{i j}
$$

where $L_{i j}=\overline{\bar{u}_{i} \bar{u}_{j}}-\bar{u}_{i} \bar{u}_{j}$ are called Leonard stresses, $C_{i j}=\overline{\bar{u}_{i} u_{j}^{\prime}}+\overline{u_{j}^{\prime} \bar{u}_{i}}$ are the cross terms, and $R_{i j}=\overline{u_{i}^{\prime} u_{j}^{\prime}}$ are Reynolds stresses. The Leonard stresses are between resolved scales and can be computed explicitly.

The cross terms represent interactions between resolved and unresolved scales, and the Reynolds stresses represent interactions within the small scales. The most popular subgrid stress (SGS) model is Smagorinsky model based on the eddy viscosity idea. The subgrid stress is written as

$$
\tau_{i j}=-2 v_{s g s} \bar{S}_{i j}
$$

where $\bar{S}_{i j}=\left(\partial \bar{u}_{i} / \partial x_{j}+\partial \bar{u}_{j} / \partial x_{i}\right) / 2$ is the resolved rate of strain tensor, and $v_{s g s}$ is eddy viscosity. Eddy viscosity is modeled as $v_{s g s}=\left(C_{s} \Delta\right)^{2}\left|\bar{S}_{i j}\right|$ where $|\bar{S}|^{2}=2 \bar{S}_{i j} \bar{S}_{i j}$ is the magnitude of the rate-of-strain tensor, $\Delta$ is the characteristic length scale, and $C_{S}$ is a dimensionless number called the Smagorinsky coefficient.

\section{SUBGRID STRESS MODEL}

Misra and Pullin [7] proposed a new structural model for subgrid stress tensor based on a stretched-vortex representation of the subgrid scales. Subgrid scale motion is modeled as stretched two dimensional vortex and the vortices are oriented randomly through the main axis. The proposed model is

$$
\tau_{i j}=\left(\delta_{i j}-e_{i}^{v} e_{j}^{v}\right) \int_{k_{c}}^{\infty} \mathscr{E}(|k|) d k
$$

where $k$ is the wave number, $\mathscr{E}(|k|)$ is energy spectrum, $k_{c}$ is cut-off number, and $\left(\delta_{i j}-e_{i}^{v} e_{j}^{v}\right)$ is the orientation tensor. Misra and Pullin [7] have approximated subgrid stress as consisting of only Reynolds stress term as $\tau_{i j}=\overline{u_{i}^{\prime} u_{j}^{\prime}}$. Leonard stress and cross stress terms have been neglected. Based on Misra and Pullin's approach, we develop a new statistical model using Çinlar stochastic velocity field [4] and its two point correlation. The energy spectrum is computed with right truncated Gamma distribution for radii of vortices. Çinlar velocity field describes features of isotrophic, stationary, and homogeneous turbulence for incompressible fluids.

\section{Subgrid Velocity Field}

Çinlar [4] has defined the basic eddy $v$ which is a deterministic velocity field on $\mathbb{R}^{2}$. Eddies of different sizes and amplitudes for $q \in Q$ where $Q=\mathbb{R}^{2} \times \mathbb{R} \times(0, \infty)$ is an index set , $x \in \mathbb{R}^{2}$ are obtained by

$$
v_{q}(x)=a v\left(\frac{x-z}{b}\right), \quad q=(z, a, b) .
$$

Let $N$ denote a Poisson random measure on the Borel sets of $\mathbb{R} \times Q$ with mean measure

$$
\mu(d t, d q) \equiv \mu(d t, d z, d a, d b)=\lambda d t d z \alpha(d a) \beta(d b)
$$


where $\lambda$ is the arrival rate per unit time-unit space, and $\alpha$ and $\beta$ are probability distributions. The arrival time $t$ of an eddy, its location $z$ in space, its amplitude $a$ as well as its radius $b$ are all random and governed by $N$.

Çağlar has generalized Çinlar velocity field [3] by introducing a dependence of the decay on the spatial variable $x$ and the index $q$ by $c_{q}(x)=c\left|\frac{x-z}{b}\right|^{2 \gamma}, q=(z, a, b)$ for each $x \in \mathbb{R}^{d}$ where $c>0$ is the decay parameter and $\gamma>0$. For $x \in \mathbb{R}^{2}, t \in \mathbb{R}$; the velocity field is given by

$$
u(x, t)=\int_{-\infty}^{t} \int_{Q} N(d s, d z, d a, d b) e^{-c|(x-z) / b|^{2 \gamma}(t-s)} a v\left(\frac{x-z}{b}\right)
$$

where $v=\left(v_{1}, v_{2}\right)$ corresponds to rotation around 0 with magnitude $m(r)$ at distance $r$ from 0 , and $m: \mathbb{R} \rightarrow \mathbb{R}_{+}$is continuous and has support $[0,1]$. Equation (3) is simply a random sum of vortices with random parameters. They arrive at Poisson points in time and decay exponentially in magnitude thereafter. The specific expressions for $v$ are $v_{1}(x)=-\frac{x_{2}}{r} m(r), \quad v_{2}(x)=\frac{x_{1}}{r} m(r)$ where $x=\left(x_{1}, x_{2}\right)$ and $r=|x| \in[0,1]$. As a rotation on $\mathbb{R}^{2}, v$ is incompressible and it vanishes outside the unit disk through the choice of $m$.

Choosing the distribution $\beta$ of $b$ as a right-truncated Gamma distribution given by

$$
\beta(d b)=\frac{b^{\theta-1} \exp (-b / \zeta)}{\Gamma_{B / \zeta}(\theta) \zeta^{\theta}} d b, \quad 0<b<B
$$

where $\theta>0$ and $\zeta>0$ are the shape and scale parameters, respectively, and $\Gamma_{B / \zeta}(\theta)$ is the incomplete Gamma function with parameter $\theta$ and integration bounds from 0 to $B / \zeta$, the energy spectrum has been computed as

$$
\mathscr{E}(|k|) \propto \frac{\lambda \mathbb{E}\left(a^{2}\right)|k|^{-\theta-4}}{c \zeta^{\theta} \Gamma_{B / \zeta}(\theta)} \int_{0}^{B|k|} e^{-b /(|k| \zeta)} b^{\theta+2} f(b) d b
$$

where

$$
\begin{array}{rl}
f(b)=\int_{0}^{1} \int_{0}^{1} & m(|z|) m(|x|) \frac{(|x||z|)^{1 / 2}}{|x|^{2 \gamma}+|z|^{2 \gamma}} \\
& (\sin (b(|x|+|z|))-\cos (b(|x|-|z|))) d|z| d|x|
\end{array}
$$

Following the vortex based SGS model (2) of Misra-Pullin [7], the parameters of the energy spectrum of Çinlar velocity field are determined from dimensional analysis and the properties of the velocity parameters in the next subsection.

\section{Parameterization of Subgrid Stress}

The Smagorinsky SGS stress model defines an eddy viscosity that is proportional to the filter width and the characteristic filtered rate of strain. Decay rate $c$ dissipates small eddies in Çinlar velocity field, so it is approached as eddy viscosity and proportional to strain rate $\left|\bar{S}_{i j}\right|$ as well. In incompressible fluid, strain rate affects eddies' shape such as stretching and deforming eddies which leads to their splitting into two or more smaller ones.

Since the strain rate brings about decomposition of eddies, we also take the radius $b$ as inversely proportional to $\left|\bar{S}_{i j}\right|$. In other words, $\left|\bar{S}_{i j}\right|$ deals with how small $b$ gets per unit time. For determining the distributional parameters of $b$, note that the mean and variance of $b$ are $\theta \zeta$ and $\theta \zeta^{2}$, respectively. Clearly, the dimension of mean radius is length $L$. Then, the dimension of the scale parameter $\zeta$ is $L$ because the shape parameter $\theta$ is dimensionless. It follows that we can model $\zeta \propto \frac{1}{\left|\bar{S}_{i j}\right|}$. While $\left|\bar{S}_{i j}\right|$ increases, both the mean and variance of $b$ decrease as implied by the formation of smaller eddies.

The arrival rate $\lambda$ is defined as the number of eddies per unit area and time. Its dimension is $[\lambda]=1 /\left(T L^{2}\right)$ where $T$ indicates time. If the strain rate $\left|\bar{S}_{i j}\right|$ increases, $\lambda$ will also increase as a consequence of formation of new eddies in subgrid scale. Therefore, $\lambda$ will be represented as $\lambda \propto \frac{\left|\bar{S}_{i j}\right|}{\Delta^{2}}$ by dimensional analysis.

Lundgren and Burgers $[1,6]$ described radial velocity as $u_{r}=-A r / 2+v_{r}(r, \theta, t)$ where $A$ is strain rate. It is assumed that the radial velocity decreases linearly in $r$ at rate $A$. Kosovic, Pullin and Samtaney [5] have taken $A$ in Lundgren energy spectrum to be $\left|\bar{S}_{i j}\right|$ and have developed subgrid models for LES of compressible turbulent flows. However, in 
Çinlar velocity field, the radial velocity magnitude, which is described by $a$, decreases exponentially in time. In the original Çinlar velocity field, the decay of $a$ is realized by the coefficient $e^{-c t}[2]$ as

$$
u(x, t)=e^{-c t} u(x, 0)+\int_{0}^{t} \int_{Q} N(d s, d z, d a, d b) e^{-c(t-s)} a v\left(\frac{x-z}{b}\right) .
$$

Although the exponential decay is more involved in the generalized model used in this paper as apparent from (3), we approximate the radial velocity decay simply by $e^{-c} a$ at an increment of unit time. Since the mean of $a$ is estimated as 0 , the variance parameter of $a$ needs to be modeled. Therefore, the variance is taken to be proportional to an exponential function of strain rate as

$$
E\left(a^{2}\right) \propto e^{-2\left|\bar{S}_{i j}\right|}
$$

in view of the approximation $c \propto\left|\bar{S}_{i j}\right|$.

Energy spectrum (4) can be rewritten using the above approximations as

$$
\mathscr{E}(|k|) \propto \frac{\left|\bar{S}_{i j}\right|^{\theta} e^{-2\left|\bar{S}_{i j}\right|}|k|^{-\theta-4}}{\Delta^{2} \Gamma_{\Delta\left|\bar{S}_{i j}\right|}(\theta)} \int_{0}^{\Delta|k|} e^{-b\left|\bar{S}_{i j}\right| /|k|} b^{\theta+2} f(b) d b
$$

where the radius of the largest eddies in dissipation range $B$ is taken equal to the grid size $\Delta$. We model the subgrid stress $\tau$ simply as proportional to the Reynolds stress, namely, $\tau_{i j} \propto \int_{k_{c}}^{\infty} \mathscr{E}(|k|) d k$. Using (5), we get

$$
\tau_{i j} \propto \frac{\left.\kappa\left|\bar{S}_{i j}\right|\right|^{\theta} e^{-2\left|\bar{S}_{i j}\right|}}{\Delta^{2} \Gamma_{\Delta\left|\bar{S}_{i j}\right|}(\theta)} \int_{k_{c}}^{\infty} \int_{0}^{\Delta|k|} e^{-b\left|\bar{S}_{i j}\right| /|k|} b^{\theta+2} f(b)|k|^{-\theta-4} d b d k .
$$

Note that we have ignored any coefficients in the modeling of the parameters $a, b, c, \lambda$ with the strain rate $\left|\bar{S}_{i j}\right|$, above. These need to be considered in the numerical solution stage, in the same way Smagorinsky coefficient is computed in LES applications.

\section{DISCUSSION}

In this study, we have modeled the parameters of energy spectrum using the resolved strain rate and developed a new subgrid model for LES of incompressible turbulent flows. This model has been based on Çinlar velocity field for small scale structures. As future work, we aim to implement our subgrid model with OPENFOAM software and validate by comparing it with other models.

\section{REFERENCES}

1. Burgers J.M., "A mathematical model illustrating the theory of turbulence". Adv. Appl. Mech. 1948; 1:171-199.

2. Çağlar, M., Piterbarg,L., Ozgokmen, T., "Parameterization of Submeso-Scale Eddy-Rich Flows using a Stochastic Velocity Model". Journal of Atmospheric and Oceanic Technology 2006;23:1745-1758.

3. Çağlar, M., "Velocity Fields with Power-Law Spectra for Modeling Turbulent Flows". Applied Mathematical Modelling 2007; 31:1934-1946.

4. Çinlar, E., "On a Random Velocity Field, Princeton University", 1993.

5. Kosovic, B., Pullin, D.I., Samtaney, R., "Subgrid-scale modeling for large-eddy simulations of compressible turbulence". Physics of Fluids 2002;14-4:1511-1522.

6. Lundgren, T. S., "Strained spiral vortex model for turbulent fine structure". Phys. Fluids 1982; 25-12:2195-2203.

7. Misra A., Pullin D. I., "A vortex-based subgrid stress model for large-eddy simulation", Phys. Fluids 1997; 9:2443-2454. 